\title{
Conceptual model of information support for taking decisions
}

\author{
Yury Nurulin $^{1 *}$, and Inga Skvortsova ${ }^{1}$ \\ 1 Peter the Great St. Petersburg Polytechnic University, Institute of Industrial Management, Economy \\ and Trade, 195251 Polytechnicheskaya st. 29, Russian Federation
}

\begin{abstract}
The modern society is characterized by qualitative changes in the volumes, composition and significance of collected and processed data. This data reflects the condition of the real and virtual world surrounding people and it is used for respective impact on the real and virtual world. All this requires theoretical generalizations that form a single conceptual platform as for managers working with the real world and for knowledge management professionals who work with the virtual world. The traditional model of taking decisions in management indirectly reflects the significance of knowledge and other elements of the virtual world for taking effective decisions which have impact on the real world. However the process of forming knowledge is not reflected in these models. Traditional models of knowledge management mainly concentrate on the internal transformation processes "data-information-knowledge-wisdom" which further are used for taking decisions.
\end{abstract}

\section{Introduction}

\subsection{Classical model of taking decisions}

Back in 1954 Peter Drucker called the process of taking effective decisions the essential function of a manager. He suggested a model of the process of taking decisions which consists of a logically connected sequence of actions aimed at finding the best decision [1].

Later this model was developed by G. Simon who suggested the conception of limited rationality [2]. He noted that though the problem has the best solution it is not always possible to find it because of limited cognitive ability of the person who takes decisions decision maker (DM). In reality DMs often don't have enough information for determining the problem, they don't see all the available alternatives and the knowledge used by them for taking decisions is not full. As a result, the decision usually leads to satisfactory but not optimal consequences. Relying on this observation Simon replaced the task of finding the best decision by the task of finding effective decisions.

Later this model was elaborated and making more detailed and it is still in the focus of the analysis of the Russian and foreign scientists [3-5].

\footnotetext{
* Corresponding author: yury.nurulin@gmail.com
} 


\subsection{Informational model}

Since the 80 -ies of the last century in the scientific literature there is a lively discussion about the nature of information and the process of cognition. A significant role in this discussion belongs to R. Ackoff who suggested a model of data transformation in 1989 [6]. This model has been called a DIKW-model in the English-language literature as an abbreviation of the English words Data, Information, Knowledge and Wisdom. The model is based on the assumption that data can be used for obtaining information, information for obtaining knowledge and knowledge - for developing wisdom. The studies where the DIKW-model is used note its main features.

1. The model has hierarchical structure which describes the processes of transformation of the essence of the lowest level of hierarchy into the essence of the top level, at this, each level adds some new properties to the previous one [7, p. 164]. Quite often this hierarchy is displayed in the form of a pyramid (Fig. 1).

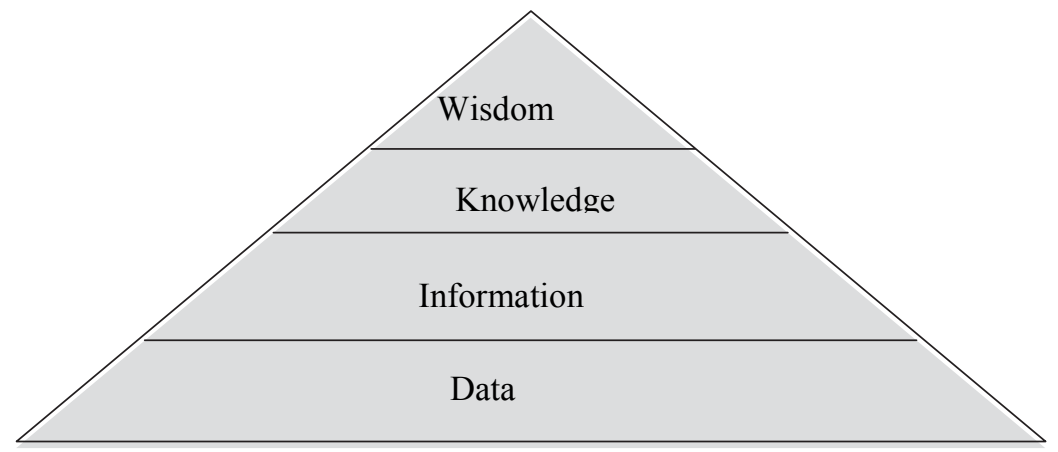

Fig. 1. Pyramid of the DIKW [7].

2. The hierarchy possesses the property of continuity both in relation to the process and in relation to the object of transformation [8].

3. There are no unified definitions for the main terms which are used in the DIKWmodel. [9].

\section{The principles of model convergence in taking decisions}

Currently both the models mentioned above are developing independently. Specialists of the information field improve methods and means for knowledge management, at this, questions of using the obtained knowledge are not the subject of analysis. Specialists working in the sphere of management develop models of taking decisions taking into account business-processes and their environment, at this, the questions of informational support for taking decisions are analyzed insufficiently. Two significant elements are indirectly present in the classic model of taking decisions: information on the condition of the observed object and its environment and also cognitive abilities of the DM which reflect his knowledge and ethical and moral standards and values. In this regard, a significant part of discussions on the nature of the process of making decisions is devoted to exploring the principles of rational behavior of a DM [10]. This reflects the general tendency: the impact of psychology on the models and methods used in economy and management increases [11]. The DM is considered as a socially active agent (an individual or a group) whose system of preferences is one of the key factors determining his behavior in the process of making decisions. However, it is necessary to note an insufficient number of works connected with exploring the influence of the informational component on the process of taking decisions, 


\subsection{Information support for taking decisions}

The indispensable link between information and making decisions was first noted in 1958 by March and Simon: processing of information and taking decisions of different levels should be considered as the key types of activity [12] for any organization.

The pyramid, as a visual reflection of the DIKW-model, shows that the number of elements at each level decreases while we move upwards through the hierarchy. Informational overload takes place when the DM receives for analysis too many elements of the lower level of the DIKW-hierarchy and that, in its turn, happens when data replaces information for describing a problem, information replaces knowledge, etc. From here it follows that there are two quite evident conclusions about the principles of information support of the decision-making process.

1. Information support of business processes should be organized according to the principles of the DIKW hierarchy. Transition to a new hierarchy level of making decisions should be accompanied by changing the information essence. It correlates well enough with the known principles of effective management which are based on the statement that the problem should be possibly solved on the level of hierarchy where it appeared.

2. Basic competencies of a manager should include the ability for effective knowledge management, the ability to acquire information from data and knowledge from information, and also the skills of using modern instruments of knowledge management.

\subsection{The models of the manager and the business-environment cooperation}

The conscious process of interplay between the human and the surrounding world can be represented as a sequence of the following stages:

$$
\text { observation } \rightarrow \text { description } \rightarrow \text { explanation } \rightarrow \text { impact (use). }
$$

At the first and the last stages of the process the subject cooperates with the real world, and the central stages belong to the virtual world which doesn't exist without the subject. The classic DIKW-model reflects the virtual world and has only indirect connections with the real world. For using in management it should be adjusted to the stages of cooperation of the subject with the real world.

In general terms, in the context of direct engineering of business "from top downward" these tasks can be represented as an iterative answer to the following sequence of questions:

$$
\text { what for } \rightarrow \text { how } \rightarrow \text { why } \rightarrow \text { what } \rightarrow \text { who } \rightarrow \text { when } \rightarrow \text { how many }
$$

In the context of the business-analysis "from top downward" in accordance with the classic model of taking decisions these tasks are transformed in the following way:

how many, where, when, who, what (happened) $\rightarrow$ why (it happened) $\rightarrow$ how (is it possible to correct it) $\rightarrow$ what for (it should be done)

Taking decisions complies with the rules of business-analysis that is why it is necessary to solve two tasks for developing an integrated model:

- put the elements of the DIKW-hierarchy and the key questions of the businessanalysis according to the classic model of taking decisions in direct correlation;

- suggest subject-oriented statements of the main elements of the DIKW-hierarchy from the perspectives of management. 


\section{The integrated model of taking decisions}

The basis for developing an integrated model is the model of the W2T-cycle which reflects cyclical pattern of cooperation of the real and virtual world through the subject: "from objects to data, information, knowledge, wisdom, services, subjects and back to objects" [14]. In this model the DIKW-hierarchy is based on the signals which come from the real world. The signals reflect the condition of the objects from the real world and transform into data through results of observation. The virtual world is connected with the real world through services (business-processes) and subjects (DM). The DM place in businessprocesses and also cooperation of the DM with the elements of the DIKW-hierarchy are not reflected in the model.

As the DM is the key element of the decision-making process the integrated model should reflect both the procedures of information support for the DM and also the basic procedures of his cooperation with the business-environment. Taking into account the fact that observations can be carried out for objects both of the real and also of the virtual world we suggest the following definition of the main categories of the DIKW-hierarchy from the perspectives of management.

Data - results of observations and measuring objects of the real and virtual world collected without context processing and available for analysis and further processing. In other words, data is a category of the virtual world which describes the real world independently from cognitive components of the observed subject. The key words in this definition are "independently from cognitive components of the observed subject". While moving upwards through the DIKW-hierarchy the results of processing become more and more dependent on cognitive capacities of the subject. That is why the hierarchy level following the data can be represented as the result of describing the world formed by the subject on the basis of context analysis of data.

Information - combination of data unified by the context for adequate description of the world. In other words, information is the description of the real of virtual world obtained by the subject on the basis of context processing of results of observation and measuring. The role of cognitive abilities of the subject in extracting information from data is relatively small.

Active discussion of the nature of knowledge is carried out in scientific literature. A number of authors underlined the indispensable link between the levels of hierarchy and defined information as simplified knowledge and, on this basis, they stated that the notions "information" and "knowledge" are synonims $[15,16]$. However, the majority of research workers note the presence of qualitative differences of essences which belong to different levels of hierarchy. Considering the nature of knowledge, Polany [17] and also Nonaka and Takeuchi and then Bennet and other research workers distinguished direct and indirect types of knowledge $[17,18]$. The bsis of this classification is a degree of formalizing knowledge. In this work [19] a break between the given classification and practical problems which are being solved by management are noted.

The analysis from literature mentioned above shows that the connections between knowledge and taking decisions are not studied in the direct form. At the same time such connections are present in implicit form because the distinguished types are associated with the key questions analyzed when taking decisions.

For building a combined model of the decision process in the work the following subject-oriented definition of knowledge is suggested.

Knowledge is a category characterized by cognitive abilities of a subject according to contextual selection, analysis and use of information for describing problems of both the virtual and the real world (why it happened). 
The interpretations of the term "knowledge" mentioned above do not ensure smooth transition to the top level of the DIKW-hierarchy from the perspectives of taking effective decisions. For eliminating this disadvantage in the work it is suggested to introduce an additional level into the DIKW-model - Understanding, as an intermediate stage of transition from knowledge to wisdom. The necessity of constructing this level is explained by the following reasons. Knowledge is the last category of the hierarchy which is focused on the past and the present whereas a number of elements of the classic model of taking decisions is focused on the future. Wisdom reflects strategic view of the consequences of a decision including those in the distant future whereas it is important to form and analyze the scenarios of the nearest future for taking decisions. Besides, a combination of moral and ethical values forms the basis of wisdom whereas the classic model of taking decisions is based on the planned condition of the object. The essence of understanding as the level of the DIKW-hierarchy consists in transforming knowledge from the past to the future through the combination of cause-effect relationship of the analyzed events. Thus, understanding is a category which characterizes the abilities of the subject to extrapolate modern tendencies on the future which are defined as a result of analyzing the events which has taken place in the virtual and real world.

The finishing stage of decision-making in the DIKW-model is wisdom. Zeleny (M. Zeleny) explains wisdom as a category which is associated with the term know-why [20]. Rowley and [20] underline that wisdom has moral, social and practical measuring (wisdom is the result of integration of knowledge with moral principles) [21].

As the highest level of abstraction wisdom characterizes the ability to "look ahead". In the Russian-language literature on the theory of decision-making it corresponds with the term "the system of preferences of a DM" to a significant extent [22]. Wisdom associates with the DM's opportunities for choosing effective alternatives in the classic model. Thus, in the combined model of taking decisions, Wisdom is a category characterizing ethical principles of a DM and his cognitive abilities to foresee technical, economic and socialethical consequences of the decisions taken Fig. 2. 


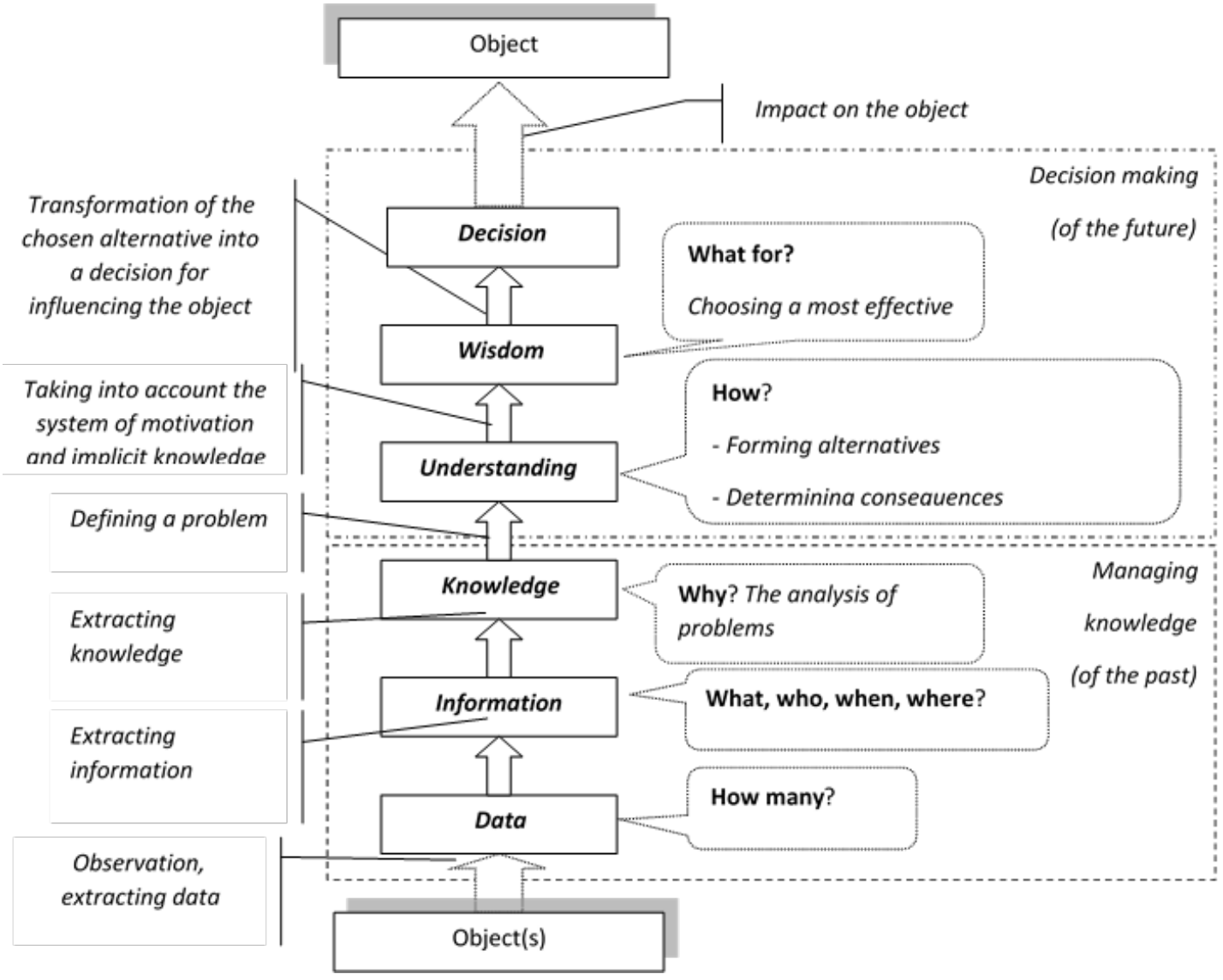

Fig. 2. The integrated model of taking managerial decisions.

We can distinguish the following basic components of the real world: material technical components (products, services, technologies); economic components (finances, organizations, business-models, market) and social components (code of behavior, jurisprudence, culture). The virtual world created by the man is also multi-component. Except data, information, knowledge it reflects the system of opinions, mentality, language, arts, entertainment, etc. Acting on the basis of wisdom DMs should directly or indirectly take into account all these components while making decisions. 


\section{Conclusion}

The suggested model has conceptual character and reflects the main approaches to organizing the processes of taking decisions from the perspectives of management and knowledge management. At the stage of strategic control it enables forming general principles of constructing business-processes oriented at taking effective managerial decisions on the basis of the DM knowledge system. At the level of tactical management it emphasizes the effective information support of the decision-making process for excluding informational overloads of the DM and providing him with necessary information. At the contextual (technical) level it underlines the necessity of using contemporary instruments for collecting and analyzing information in the process of taking decisions, extracting information from them and generating knowledge. Integration of the considered approaches forms the necessary methodological base for forming the future economy structure.

\section{References}

1. P. Drucker, The Practice of Management (Harper \& Brothers, New York, 1954)

2. H. Simon, Administrative Behavior (The Free Press, New York, 1976)

3. V. G. Smirnova et al., Organization and its business environment: 17-module environment for managers "Organization development control" Module 2 (INFRA-M, Moscow, 2000)

4. F. Adam, Journal of Decision Systems, 21 (2) (2012)

5. A Chaabounia, I. Yahiab, Journal of Decision Systems, 23(3) (2014)

6. R. Ackoff, Journal of Applies Systems Analysis, 16 (1989)

7. J. Rowley, Journal of Information Science, 33 (2007)

8. D. Pan, Computing in Science and Engineering, 5 (2010)

9. S. Baškarada, A. Koronios, Australasian Journal of Information Systems', 18 (1) (2013)

10. J. Basel, R. Jörn, European Management Journal, 31 (2013)

11. S. Della Vigna, Journal of Economic Literature, 47 (2) (2009)

12. J. March, H. Simon, Organizations (Wiley and Sons, New York, 1993)

13. P. Hemp, Harvard Business Review, 87 (9) (2009)

14. N Zhong, J Ma, R. Huang, J. Liu, Y. Yao, Y. Zhang, \& J. Chen, Journal of Supercomputing, 64 (3) (2013)

15. M Swigon, Journal of Information Science, 39 (6) (2013)

16. M. Fricke, J. Inf. Sci, 35(2) (2009)

17. M. Polanyi, Personal knowledge: Towards a post-critical philosophy (Routledge, London, 1958)

18. D. Bennet, A. Bennet, J. Inf. Know. Man. Syst, 38 (2008)

19. P. Hulme, J. Appl. Ecol., 51 (2014)

20. J. Rowley, F. Slack, J. Inf. Sci., 35 (1) (2009)

21. M. Zeleny, Human Syst. Man., 7 (1) (1987)

22. Z. P. Rumyantseva et al., General organization control: principles and processes: $17-$ module program for managers "Organization development control". Module 3 (INFRA-M, Moscow, 2000) 